\title{
Wave-function localization in reciprocal space
}

\author{
Iannuzzi, Marcella ; Parrinello, Michele
}

\begin{abstract}
The localization of wave functions in real space is known to be extremely helpful in the investigation of the electronic properties and in the development of $\mathrm{O}(\mathrm{N})$ methods. However, maximally localized wave functions in real space do not provide a good representation of the Bloch orbitals of a metallic system, where the localization procedure is very inefficient and of little use. On the other hand, in metals, it appears more natural to localize the wave functions in reciprocal space. In this work we propose a spread functional, which needs to be minimized in order to obtain maximally localized wave functions in reciprocal space and illustrate an efficient iterative minimization procedure. We also discuss the application of the method to some metallic systems and demonstrate that in this case the localized wave functions have features that can be useful for the analysis of electronic properties.
\end{abstract}

DOI: https://doi.org/10.1103/PhysRevB.66.155209

Posted at the Zurich Open Repository and Archive, University of Zurich

ZORA URL: https://doi.org/10.5167/uzh-138234

Journal Article

Published Version

Originally published at:

Iannuzzi, Marcella; Parrinello, Michele (2002). Wave-function localization in reciprocal space. Physical review. B, 66(15):155209.

DOI: https://doi.org/10.1103/PhysRevB.66.155209 


\title{
Wave-function localization in reciprocal space
}

\author{
Marcella Iannuzzi and Michele Parrinello \\ CSCS - Centro Svizzero di Calcolo Scientifico, via Cantonale, CH-6928 Manno, Switzerland \\ and Physical Chemistry ETH, Hönggerberg HCI, CH-8093 Zurich, Switzerland
}

(Received 27 June 2002; revised manuscript received 26 August 2002; published 30 October 2002)

\begin{abstract}
The localization of wave functions in real space is known to be extremely helpful in the investigation of the electronic properties and in the development of $O(N)$ methods. However, maximally localized wave functions in real space do not provide a good representation of the Bloch orbitals of a metallic system, where the localization procedure is very inefficient and of little use. On the other hand, in metals, it appears more natural to localize the wave functions in reciprocal space. In this work we propose a spread functional, which needs to be minimized in order to obtain maximally localized wave functions in reciprocal space and illustrate an efficient iterative minimization procedure. We also discuss the application of the method to some metallic systems and demonstrate that in this case the localized wave functions have features that can be useful for the analysis of electronic properties.
\end{abstract}

DOI: $10.1103 /$ PhysRevB.66.155209

PACS number(s): 71.10.-w, 71.15.Ap

\section{INTRODUCTION}

It is well recognized that Wannier functions are an extremely useful tool for understanding the chemical properties of condensed systems. ${ }^{1}$ They are derived from the KohnSham (KS) orbitals via unitary transformation which leads to localized wave functions. ${ }^{2}$ Very recently Marzari and Vanderbilt proposed an efficient and well defined way of calculating the Wannier orbitals ${ }^{3}$ and nowadays such calculations are routinely performed. ${ }^{4}$ The possibility of localizing the KS orbitals provides theoretical underpinning of several $O(N)$ methods. ${ }^{5}$ For systems that have a finite energy gap it is generally accepted that the Wannier functions decay exponentially, ${ }^{6}$ with a characteristic decay length that depends on the gap. For metals, however, the localization is expected to lead to an algebraic decay, which, of course, limits the usefulness of the Wannier functions and prevents the application of most $O(N)$ methods. The possibility of improving the localization of the wave functions, i.e., the sparseness of the density matrix, in real and Fourier space, by using the localization properties of appropriate basis sets, ${ }^{7}$ has been considered by Goedecker et al. ${ }^{8}$ and by Niklasson et al. ${ }^{9}$ for model systems.

Here we explore the possibility of localizing the wave functions in the reciprocal space, in the framework of the general plane waves basis set. We devise a procedure that makes this possible and we find that in metals such as $\mathrm{Na}$ the wave functions can be very efficiently localized. In contrast in semiconducting materials such as $\mathrm{Si}$, this is less useful. In either case the localization procedure provides useful insight into the properties of wave functions.

The idea of maximally localized orbitals in reciprocal space is to work with functions of finite support, i.e., functions that are nonzero only in limited regions. Such a representation would potentially improve the computational efficiency of frequently required operations such as scalar products. Having in mind applications to very large systems such as those that are affordable now or in the near future, we will consider only systems where the Brillouin Zone (BZ) is sampled in the $\Gamma$ point of a large supercell. ${ }^{10}$

\section{WAVE FUNCTION LOCALIZATION IN REAL SPACE}

We recall here the procedure to generate maximally localized Wannier functions $\left\{W_{n}(\mathbf{r})\right\}$. These are obtained via the unitary transformation $U$ in the space of the $N$ occupied KS orbitals $\left\{\psi_{n}(\mathbf{r})\right\}^{2}$

$$
\left|W_{i}\right\rangle=\sum_{j=1}^{N} U_{i j}\left|\psi_{j}\right\rangle .
$$

$U$ is uniquely determined by imposing the minimization of the spread functional $\Omega$,

$$
\Omega=\sum_{i=1}^{N}\left[\sum_{\alpha}\left\langle W_{i}\left|r_{\alpha}^{2}\right| W_{i}\right\rangle-\left\langle W_{i}\left|r_{\alpha}\right| W_{i}\right\rangle^{2}\right],
$$

where $\mathbf{r}$ is the position operator and $\alpha$ runs over the Cartesian coordinates. The calculation of $\Omega$ is straightforward in the case of a finite system, and in this case the $\left\{W_{n}(\mathbf{r})\right\}$ coincide with the Boys' orbitals. ${ }^{11}$ Under periodic boundary conditions, on the other hand, the position operator $\mathbf{r}$ is ill defined and another formalism has to be adopted. We adopt here the approach of Resta ${ }^{12}$ and define the spread functional

$$
\Omega=\frac{1}{(2 \pi)^{2}} \ln |z|^{2},
$$

where $z$ is a dimensionless complex number given by the volume integral

$$
z=\int_{L^{3}} d \mathbf{r} e^{i(2 \pi / L)(x+y+z)}|\psi(\mathbf{r})|^{2},
$$

where a cubic lattice is assumed, whose dimension is $L$.

Starting from Resta's functional, Berghold et al. have studied an appropriate generalization of the spread formula for arbitrary simulation cells. ${ }^{13}$ For an orthorhombic lattice, the functional that they propose is 


$$
\Omega=\frac{2}{(2 \pi)^{2}} \sum_{n} \sum_{\alpha} \omega_{\alpha}\left(1-\left|\int_{V} \psi_{n}^{*}(\mathbf{r}) e^{i \mathbf{G}_{\alpha} \cdot \mathbf{r}} \psi_{n}(\mathbf{r}) d \mathbf{r}\right|^{2}\right),
$$

where the first sum is over all the occupied states, $\mathbf{G}_{\alpha}$ are the smallest reciprocal vectors along the reciprocal primitive vectors $\left(\mathbf{b}_{1}, \mathbf{b}_{2}, \mathbf{b}_{3}\right)$, and $\omega_{\alpha}$ are appropriate weights, chosen according to the rule used by Silvestrelli ${ }^{14}$ and by Marzari and Vanderbilt. ${ }^{3}$ Let us now consider a wave function expanded in plane waves (PW's), as is usually done in ab initio molecular dynamics,

$$
\psi_{n}(\mathbf{r})=\sum_{\mathbf{G}} c_{n}(\mathbf{G}) e^{i(\mathbf{G} \cdot \mathbf{r})} .
$$

In this case the integral in Eq. (5) is calculated as the sum over the mesh in real space, defined by the $N_{1} \times N_{2} \times N_{3}$ grid points

$$
\mathbf{r}_{i}=\frac{i_{1}}{N_{1}} \mathbf{a}_{1}+\frac{i_{2}}{N_{2}} \mathbf{a}_{2}+\frac{i_{3}}{N_{3}} \mathbf{a}_{3},
$$

where $\left\{\mathbf{a}_{\alpha}\right\}$ denote the direct lattice vectors, the integer numbers $i_{\alpha}$ are taken between 1 and $N_{\alpha}$ and the spread functional becomes

$$
\Omega=\sum_{n} \sum_{\alpha}\left(1-\left|\sum_{\mathbf{r}_{i}} \psi_{n}^{*}\left(\mathbf{r}_{i}\right) e^{i \mathbf{G}_{\alpha} \cdot \mathbf{r}_{i}} \psi_{n}\left(\mathbf{r}_{i}\right)\right|^{2}\right) .
$$

The $N_{\alpha}$ are an estimate for the size of the PW basis set; they are defined in terms of the cutoff energy $E_{\text {cut }}$ and the simulation cell's volume $V$, through

$$
N_{\mathrm{PW}}=N_{1} \times N_{2} \times N_{3}=\frac{\sqrt{2}}{3 \pi^{2}} V E_{\mathrm{cut}}^{3 / 2} .
$$

\section{THEORY}

In the present work we transfer the idea of wave function localization, as reported above, from real space to its dual space, the reciprocal or Fourier space. In complete analogy to the spread in direct space [Eq. (8)], a spread functional can be defined in the Fourier space, describing the extent to which the Fourier transform of an electronic wave function is scattered in reciprocal space. We write the $G$-space spread functional by constructing the dual version of Eq. (8) as

$$
\begin{aligned}
F & =\sum_{n} \sum_{\alpha}\left(1-\left|\sum_{\mathbf{G}} c_{n}^{*}(\mathbf{G}) e^{i\left(\mathbf{G} \cdot \mathbf{r}_{\alpha}^{0}\right)} c_{n}(\mathbf{G})\right|^{2}\right) \\
& =\sum_{n} \sum_{\alpha}\left(1-\left|S_{\alpha n}\right|^{2}\right) .
\end{aligned}
$$

Here, $\mathbf{r}_{\alpha}^{0}$ are fixed vectors along the primitive lattice vectors $\left(\mathbf{a}_{1}, \mathbf{a}_{2}, \mathbf{a}_{3}\right)$, whereas the sum $S_{\alpha n}$ is the diagonal element of the operator $\hat{S}_{\alpha}=e^{i \mathbf{G} \cdot \mathbf{r}_{\alpha}^{0}}$. This functional clearly has the right properties, since for a function which is fully delocalized all the $\left|c_{n}(\mathbf{G})\right|$ values are equal and the the sum $S_{\alpha n}$ vanishes. However, if the orbitals are plane waves, which corresponds to the maximum possible localization, $\left|S_{\alpha n}\right|=1$ and the contribution of the orbital to the total spread is zero.

In analogy to the problem of the localization of the orbitals in real space, we look for the unitary transformation of the orbitals $U$ such that

$$
\tilde{c}_{n}(\mathbf{G})=\sum_{m} U_{n m} c_{m}(\mathbf{G}),
$$

which minimizes the spread functional $F$. Therefore we want to solve the set of $N^{2}$ equations

$$
\frac{\partial F}{\partial U_{m n}}+\text { constraints }=0,
$$

where the constraint term is required in order to enforce the unitarity of the matrix $U$. Several methods for imposing the unitarity constraint have been described in Ref. 13. Here we take a different approach. We represent the unitary matrix in terms of its $N$-dimensional column vectors $\left|U_{n}\right\rangle n=1 \cdots N$ as

$$
\left|U_{n}\right\rangle \doteq\left(\begin{array}{c}
U_{1 n} \\
\vdots \\
U_{N n}
\end{array}\right) .
$$

By using this simple bra-ket notation, we can write the spread functional in terms of the transformed orbitals as

$$
F=\sum_{n}\left\{\sum_{\alpha}\left[1-\left\langle U_{n}\left|\hat{S}_{\alpha}\right| U_{n}\right\rangle \cdot\left(\left\langle U_{n}\left|\hat{S}_{\alpha}\right| U_{n}\right\rangle\right)^{*}\right]\right\}
$$

Moreover we realize that the condition of unitarity is equivalent to the orthonormality condition $\left\langle U_{m} \mid U_{n}\right\rangle=\delta_{m n}$, as can be easily verified. We can now borrow from the CarParrinello scheme ${ }^{16}$ and impose the orthonormality of the $\left|U_{n}\right\rangle$ via Lagrange parameters. The expression of the derivative of the functional $F$ which takes into account the $\left|U_{n}\right\rangle$ orthonormality becomes

$$
\begin{aligned}
\frac{\delta \widetilde{F}}{\delta\left\langle U_{n}\right|}= & -\sum_{\alpha}\left[\hat{S}_{\alpha}\left|U_{n}\right\rangle \cdot\left(\left\langle U_{n}\left|\hat{S}_{\alpha}\right| U_{n}\right\rangle\right)^{*}\right. \\
& \left.+\left\langle U_{n}\left|\hat{S}_{\alpha}\right| U_{n}\right\rangle \cdot \hat{S}_{\alpha}^{\dagger}\left|U_{n}\right\rangle\right] \\
& \left.+\sum_{l} \Lambda_{n l} \Delta \mid U_{l}(t)\right],
\end{aligned}
$$

where $\Lambda_{n l}$ are Lagrange multipliers to be determined with standard iterative methods. In practice we optimize $F$ using a steepest descent algorithm guided by the forces in Eq. (15), where the integration time step is determined by a minimum search along the direction of the gradient. This iterative localization algorithm has been implemented in the CPMD code, which is based on a plane wave expansion of the electronic orbitals. ${ }^{15}$

As a final remark for this section we note that the KS orbitals calculated in $\Gamma$ are commonly represented as real wave functions in direct space and their Fourier coefficients satisfy the symmetry condition $c_{n}(-\mathbf{G})=c_{n}^{*}(\mathbf{G})$, which pre- 


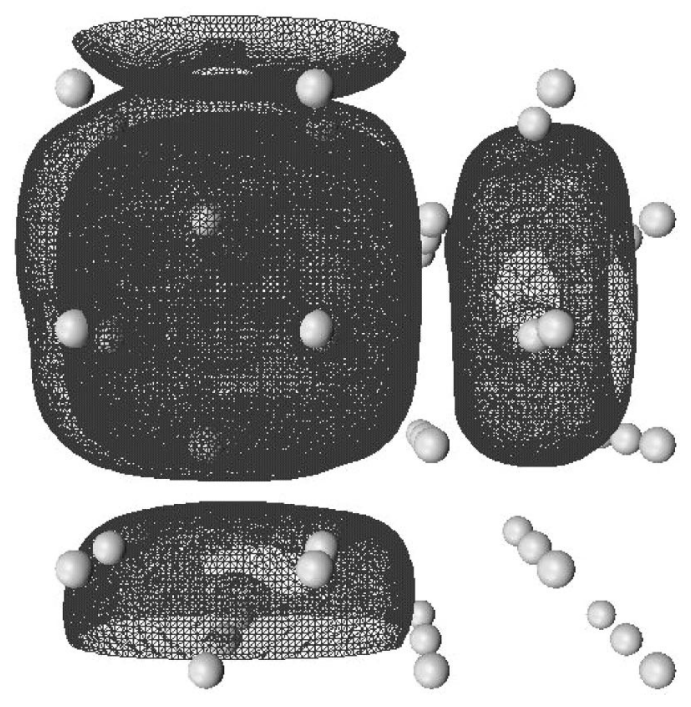

FIG. 1. Maximally localized Wannier orbital in the Na simulation cell, as obtained by the wave function localization in real space. The level surface has been cut at $10 \%$ of the maximum peak of $|\psi(\mathbf{r})|$.

vents an optimal localization in $G$ space. Therefore we relax the restriction to real orbitals and allow the $G$ space centers of the orbitals

$$
\left\langle G_{\alpha}\right\rangle_{n}=\sum_{\mathbf{G}}\left|c_{n}(G)\right|^{2} G_{\alpha}, \quad \alpha=x, y, z
$$

to be different from zero.

\section{RESULTS}

We illustrate our method with two examples, one being sodium, solid and liquid, and the other solid aluminum. For $\mathrm{Na}$ we take a cubic supercell of length $L=3 a$, where $a$ $=4.23 \AA$ is the lattice constant. The cell contains 54 atoms and $3 \times 3 \times 3$ unit cells. Due to spin degeneracy we consider 27 electronic states. For the wave function optimization we used a Goedecker pseudopotential. ${ }^{17}$

If we use the real space localization procedure for $\mathrm{Na}$ wave functions, we find that the resulting Wannier orbitals are only modestly localized, and produce a $20 \%$ reduction of the total spread with respect to the canonical KS orbitals. The probability density $|\psi(\mathbf{r})|$ of a resulting Wannier function is shown in Fig. 1. Visibly, it is spread all over the supercell. This is not surprising since it reflects the metallic nature of $\mathrm{Na}$.

Much more efficient is the localization in $G$ space, where we find a $75 \%$ reduction in spread. Within our numerical accuracy, the localized wave functions $W(\mathbf{G})$ appear to be very similar to one another. The centers of the $W(\mathbf{G})$, as defined in Eq. 16, occupy the reciprocal lattice positions

$$
\mathbf{k}_{j}=\frac{2 \pi}{L}\left(j_{1}, j_{2}, j_{3}\right), j_{\alpha}=-1,1 .
$$

Therefore, in this case we can label the localized wave function with the corresponding $\mathbf{k}_{j}$ vector $W_{\mathbf{k}_{j}}(\mathbf{G})$. The probabil-

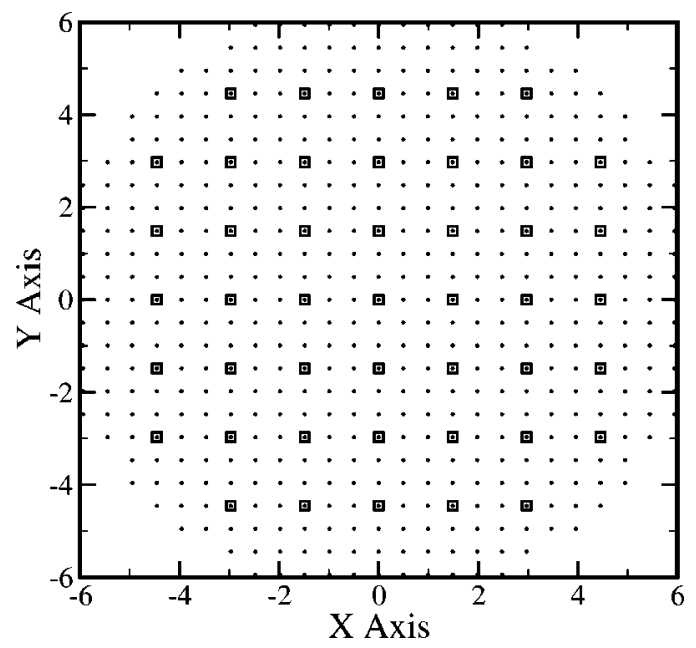

FIG. 2. The black dots denote the grid in $G$ space, whose period is $2 \pi / L$, whereas the squares are the nonzero Fourier coefficients of the $W_{0}(\mathbf{G})$ centered in the origin.

ity density is maximum at the wave function center, where $\left|W_{\mathbf{k}_{j}}\left(\mathbf{k}_{j}\right)\right|=0.98$. This reflects the quasifree electron behavior of $\mathrm{Na}$. Of course the chemistry information is contained in the remaining $2 \%$.

A noteworthy property of the $W_{\mathbf{k}_{j}}(\mathbf{G})$ is that they are different from zero only at the lattice positions

$$
\mathbf{G}=\mathbf{k}_{j}+\frac{2 \pi}{a}(l, m, n) .
$$

In Fig. 2, for example, we show the nonzero coefficients of the $W_{0}(\mathbf{G})$, which is centered in the origin. As a consequence, the probability distribution of the Fourier transform of $W_{\mathbf{k}_{j}}(\mathbf{G})$

$$
\begin{aligned}
\widetilde{W}_{\mathbf{k}_{j}}\left(\mathbf{r}_{i}\right)= & \sum_{l m n} W_{\mathbf{k}_{j}}\left(\mathbf{k}_{j}+\frac{2 \pi}{a}(l, m, n)\right) \\
& \times e^{i \mathbf{k}_{j} \cdot \mathbf{r}_{i}} e^{i(2 \pi L / a)\left(l \cdot i_{1}+m \cdot i_{2}+n \cdot i_{3}\right)}
\end{aligned}
$$

has the periodicity of the $\mathrm{Na}$ direct lattice. In addition we stress that the wave functions can be exactly superimposed on one another by simple symmetry operations; for instance, the coefficients of $W_{\mathbf{k}_{j}}(\mathbf{G})$ are related to those of $W_{-\mathbf{k}_{j}}(\mathbf{G})$ by an inversion with respect to the origin. In order to make contact with the ordinary description of the electronic state of a periodic system, we note that the vectors $\mathbf{k}_{j}$ are the BZ vectors consistent with the periodicity of the supercell. Therefore the $G$ localized wave functions in real space have a Bloch-like expression of the type

$$
\psi(\mathbf{r})=e^{i \mathbf{k} \cdot \mathbf{r}} u_{\mathbf{k}}(\mathbf{r})
$$

One can quantify the overlap between different wave functions via the functional

$$
O\left[\left\{\psi_{n}\right\}\right]=\frac{2}{N(N-1)} \sum_{n>m}\left(\sum_{\mathbf{G}}\left|c_{n}(\mathbf{G})\right|^{2}\left|c_{m}(\mathbf{G})\right|^{2}\right) .
$$


TABLE I. In the first column we indicate the relative reduction in total spread. The second and third columns report the numerical wave function overlap, before $(O)$ and $\operatorname{after}(\widetilde{O})$ the rotation of the orbitals, according to the definition given in Eq. (21).

\begin{tabular}{lccc}
\hline \hline \multicolumn{1}{c}{ Sample } & $F_{0}-F_{\text {Loc }} \%$ & $O\left[\left\{\psi_{n}\right\}\right]$ & $\widetilde{O}\left[\left\{\psi_{n}\right\}\right]$ \\
\hline Silicon & 30.4 & $2.00 \times 10^{-3}$ & $4.15 \times 10^{-4}$ \\
Graphite & 19.9 & $2.41 \times 10^{-3}$ & $0.60 \times 10^{-3}$ \\
Sodium & 75.2 & $2.77 \times 10^{-2}$ & $1.59 \times 10^{-10}$ \\
Liquid Sodium & 73.5 & $2.94 \times 10^{-2}$ & $8.61 \times 10^{-6}$ \\
Aluminum & 74.7 & $5.21 \times 10^{-3}$ & $2.14 \times 10^{-6}$ \\
\hline \hline
\end{tabular}

In the case of solid $\mathrm{Na}$, the $W_{\mathbf{k}_{j}}(\mathbf{G})$ are different from zero at different points of the reciprocal lattice and $O\left[\left\{\psi_{n}\right\}\right]$ is zero, as reported in Table I.

In order to take into account the possible influence of the pseudopotential on these results, we reproduced the same calculations by using a Bachelet-Hamann-Schlüter pseudopotential. ${ }^{18}$ In this case, the localization procedure gives a reduction in the total spread about $81 \%$ and the overlap $O\left[\left\{\psi_{n}\right\}\right]$ decreases from the initial value $2.8 \times 10^{-2}$ to $3.5 \times 10^{-15}$, thus confirming the behavior observed with the Goedecker pseudopotential.

We now consider the more interesting case of liquid $\mathrm{Na}$, which also has a metallic nature. The atomic configuration for the liquid sample is obtained from $a b$ initio molecular dynamics by melting the solid $\mathrm{Na}$ through first slowly raising the temperature to $5000 \mathrm{~K}$ and then bringing it down to 300 K. In Fig. 3 we compare the pair correlation function $g(r)$, as calculated in the liquid at equilibrium at $300 \mathrm{~K}$, to the $g(r)$ of the Na cubic lattice.

Starting from this atomic configuration, we first calculate the KS eigenstates in $\Gamma$ only and then apply the algorithm for the spread minimization in $G$ space. In analogy with the case of solid $\mathrm{Na}$, we obtain a large reduction in the total spread (73\%) and an effective localization of the wave functions, whose centers form the same cubic pattern as described for solid Na. The probability densities $\left|W_{\mathbf{k}_{j}}(\mathbf{G})\right|$ have a free electronlike behavior, since they show peaks as large as 0.95 ,

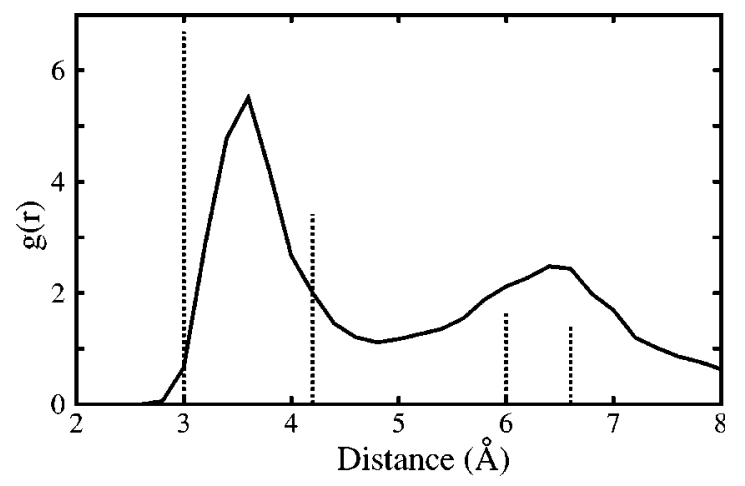

FIG. 3. Pair correlation function calculated in the equilibrated liquid sample at $300 \mathrm{~K}$. As a reference, the pair correlation function obtained in the perfect $\mathrm{Na}$ cubic lattice is also reported (dashed lines). in a single $G$ coefficient, the center of the wave function. There are, however, significant differences since now the $G$ vectors at which the $W_{\mathbf{k}_{j}}(\mathbf{G})$ are different from zero are not of the type of Eq. (18). Of course one can still write the wave functions in a Bloch-like manner, but now the $\widetilde{W}_{\mathbf{k}_{j}}\left(\mathbf{r}_{i}\right)$ do not have the lattice periodicity. Although we can distinguish a one-to-one correspondence between the $W_{\mathbf{k}_{j}}(\mathbf{G})$ and the $\mathbf{k}_{j}$ points given in Eq. (17), there are no simple symmetry operations that allow the orbitals to be superimposed on one another. It is not possible to evince any periodic pattern in the Fourier coefficient distributions. This is due to the disordered atomic structure of the liquid sample and the lack of periodic features in the supercell. Nevertheless, also in the liquid case, the localization procedure produces a separation among the wave function supports in $G$ space, such that their overlap is decreased by some orders of magnitude (Table I), according to the definition given in Eq. (21).

Before discussing the more complicated case of $\mathrm{Al}$, it is useful to make the following remarks. In a $\Gamma$ only description, the $\mathrm{BZ}$ is made up of vectors of the type

$$
\mathbf{k}_{j}=2 \pi\left(\frac{j_{1}}{L_{1}}, \frac{j_{2}}{L_{2}}, \frac{j_{2}}{L_{2}}\right) \text {, }
$$

where $L_{1}, L_{2}$, and $L_{3}$ are length of the supercell sides. For a cubic lattice such vectors can be ordered in shells of identical modulus squares, which contain the following numbers of vectors $1,6,12,8,6,24,24,12, \ldots$. In the cubic supercell of the type used here, there are 27 spin-paired states. Therefore the $\mathrm{k}$-vectors that belong to the lowest four shells can be exhausted by the one-to-one association to the electronic states. This leads to an optimal localization of the orbitals.

The situation for $\mathrm{Al}$ is different, where it is not possible to separate the shells that are fully occupied from the nonoccupied ones. Searching among many possible supercells compatible with the fcc lattice, we have chosen an orthorhombic body centered unit cell of size $a_{1}=a / \sqrt{2}, a_{2}=a / \sqrt{2}$, and $a_{3}=a$, where $a=4.05 \AA$ is the fcc lattice constant. If we repeat this cell $3 \times 5 \times 3$ times we can accommodate 90 atoms, giving 135 electronic states. The corresponding shell structure in reciprocal space is described in Fig. 4, where the number of grid points contained in the first 44 shells is indicated by the columns. The shaded region in each column indicates how many orbitals are actually localized in the corresponding shell after the spread minimization. If Al were a perfect free-electron system, we would have 131 states localized in fully occupied shells and 33 filled columns, while the remaining four states would be distributed in the next shell of eight vectors. In reality, things are slightly more complex, as we shall show below.

The localization algorithm gives a satisfactory spread minimization in $G$ space $(74 \%)$, through the transformation of the $\mathrm{Al} \mathrm{KS}$ states into the maximally localized $W(\mathbf{G})$. However, the localized wave functions are not all similar to one another. Only the first 119 states show the same behavior as the one described in the case of Na. Their wave function's centers occupy the reciprocal lattice positions 


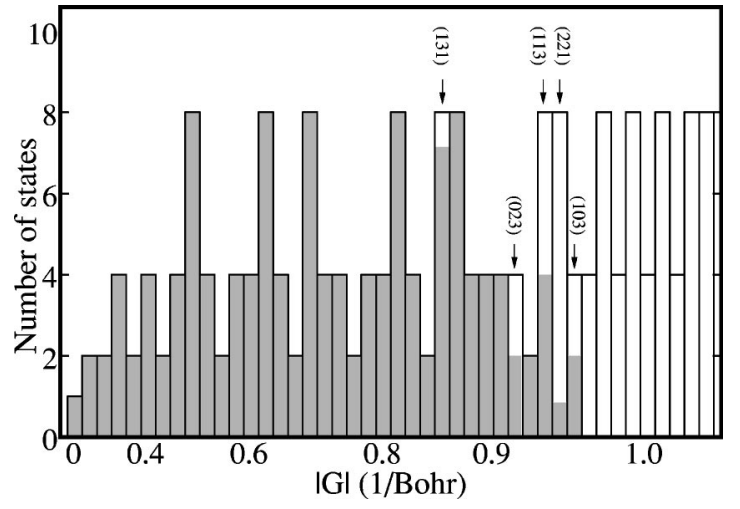

FIG. 4. Number of grid points contained in the $G$-space shells for the orthorhombic Al supercell, generated by replicating the unit cell $3 \times 5 \times 3$ times. The shaded regions indicate how many orbitals are localized in each shell.

$$
\mathbf{k}_{j}=2 \pi\left(\frac{j_{1}}{L_{1}}, \frac{j_{2}}{L_{2}}, \frac{j_{2}}{L_{2}}\right)
$$

and the probability density maximum is also localized in that single point, $\left|W_{\mathbf{k}_{j}}\left(\mathbf{k}_{j}\right)\right|=0.98$. The shells constituted by these vectors are those represented as fully occupied in Fig. 4.

Among the remaining 16 wave functions, eight have the main component as large as 0.91 in the $\{(131)\}$ shell (i.e., $j_{1}=\{1,-1\}, j_{2}=\{3,-3\}$, and $j_{3}=\{1,-1\}$ ), and a second important component with norm equal to 0.079 in the outer $\{(221)\}$ shell. Therefore the $\{(131)\}$ cannot be considered as fully occupied and the corresponding eight wave function centers do not sit exactly on the grid's points, as shown in Fig. 5. The last eight $W(\mathbf{G})$ are equally divided among two main Fourier components, relative to two opposite vectors belonging to one of the following shells: $\{(103)\},\{(023)\}$, or $\{(113)\}$. Since each $W(\mathbf{G})$ occupies two vectors in these

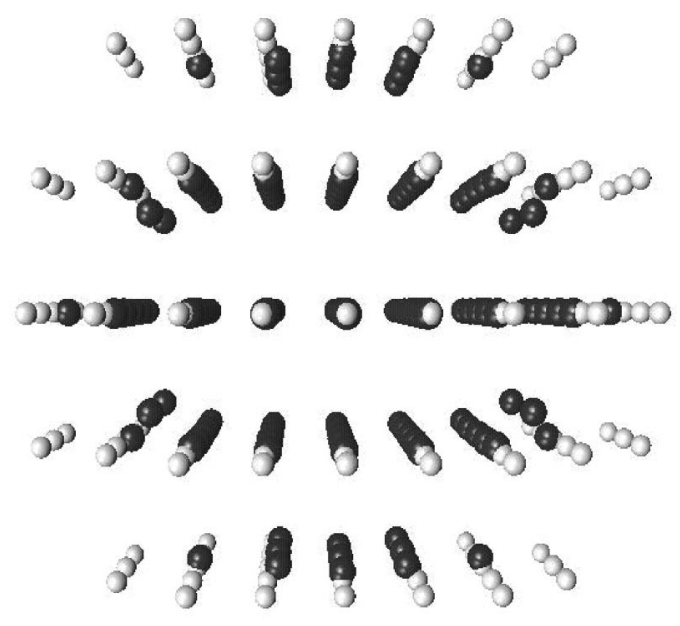

FIG. 5. The centers of the Al localized $W(\mathbf{G})$ (black spheres) are displayed inside the $G$-space grid (white spheres). 8 out of the 135 orbitals have centers displaced with respect to the grid, but close to the points of the $\{(131)\}$ shell. 8 more centers are superimposed on already occupied points, due to the symmetric distribution of the corresponding $|W F(\mathbf{G})|$ among vectors belonging to the shells $\{(103)\},\{(023)\}$, or $\{(113)\}$. shells, they turn out to be occupied by half the number of orbitals, with respect to the number of points they contain, as shown in Fig. 4. On the other hand, due to the symmetric distribution of these orbitals inside the shell they occupy, the $W(\mathbf{G})$ centers are superimposed on already occupied vectors of shells with a lower radius. All the last 16 orbitals are less efficiently localized than the first 119 . They can be seen as the linear combination of two Bloch functions of the type in Eq. (20), and therefore they should be labeled by 2 vectors $\mathbf{k}_{j}$. This peculiar behavior of the orbitals which are higher in energy can be attributed to the fact that they are localized in the region where the Fermi surface crosses the $G$-space shell's boundaries. Since in the case of Al the Fermi surface is not spherical, it is not possible to obtain a one-to-one correspondence between orbitals and $k$ vectors.

Nevertheless all the probability densities are periodically distributed and are different from zero only at

$$
\mathbf{G}=\mathbf{k}_{j}+2 \pi\left(\frac{l}{a_{1}}, \frac{m}{a_{2}}, \frac{n}{a_{3}}\right)
$$

This is enough to largely reduce the wave function's overlap in $G$ space, as reported in Table I, although the separation is less efficient than in the case of solid $\mathrm{Na}$.

\section{CONCLUSIONS}

The linearly scaling methods have not been widely used in density-functional-type calculations, because the required large number of basis functions makes it difficult to obtain sparse density matrices. We have described the theory, implementation and some applications of a successful procedure for localizing wave functions in $G$ space. The calculation of the overlap $O\left[\left\{\psi_{n}\right\}\right]$ in the reciprocal space, as reported in Table I, gives an estimation of the sparseness of the density matrix after the localization procedure. It shows that also with a conventional basis set it is possible to achieve the required localization properties that make the density matrix significantly more sparse. However the localization pattern appears to be generally rather complex, and its possible application to $O(N)$ methods challenging. We also considered the possibility of a combined localization, in real and reciprocal space, that would be the optimal condition for the application of $O(N)$ methods. As expected, the simultaneous spread minimizations, in the two spaces, compete against one another and in general the results are far from satisfactory. An exception is the case of graphite. Here, when the combined localization is required, the electrons are localized in planes perpendicular to $z$ in the real space, whereas they remain widely delocalized in the $x$ and $y$ dimensions. In the reciprocal space this corresponds to wave functions that are localized in $x^{\prime}$ and $y^{\prime}$ and are spread along the third axis.

In addition, the localization procedure illuminates many features of metallic chemistry. For instance, in liquid $\mathrm{Na}$, it allows the system to be described by electronic states that are a generalization of the Bloch orbitals. These new states can be used to describe and even quantify the free-electron character of a system. 
${ }^{1}$ G.H. Wannier, Phys. Rev. 52, 191 (1937).

${ }^{2}$ E. Blount, in Solid State Physics, edited by F. Seitz and C. Turnbull (Academic, New York, 1962), Vol. 13, p. 305.

${ }^{3}$ N. Marzari and D. Vanderbilt, Phys. Rev. B 56, 12847 (1997).

${ }^{4}$ PL. Silvestrelli and M. Parrinello, J. Chem. Phys. 111, 3572 (1999); AH. Romero, PL. Silvestrelli, and M. Parrinello, ibid. 115, 115 (2001).

${ }^{5}$ S. Goedecker, Rev. Mod. Phys. 71, 1085 (1999).

${ }^{6}$ W. Kohn, Phys. Rev. 115, 809 (1959).

${ }^{7}$ I. Daubechies, Ten Lectures on Wavelets (SIAM, Philadelphia, 1992).

${ }^{8}$ S. Goedecker and O. Ivanov, Phys. Rev. B 59, 7270 (1999).

${ }^{9}$ A.M.N. Niklasson, C.J. Tymczak, and H. Röder, Phys. Rev. B (to be published).

${ }^{10}$ N.W. Ashcroft and N.D. Mermin, Solid State Physics (Holt Saun- ders, Tokyo, 1976).

${ }^{11}$ S.F. Boys, Rev. Mod. Phys. 32, 296 (1960).

${ }^{12}$ R. Resta, Rev. Mod. Phys. 66, 899 (1994); Phys. Rev. Lett. 80, 1800 (1998).

${ }^{13}$ G. Berghold, C.J. Mundy, A.H. Romero, J. Hutter, and M. Parrinello, Phys. Rev. B 61, 10040 (2000).

${ }^{14}$ P.L. Silvestrelli, Phys. Rev. B 59, 9703 (1999).

${ }^{15}$ J. Hutter et al., CPMD V3.5 Copyright IBM Corp. 1990-2001, Copyright MPI fuer Festkoerperforschung Stuttgart 1997-2001.

${ }^{16}$ R. Car and M. Parrinello, Phys. Rev. Lett. 55, 2471 (1985).

${ }^{17}$ S. Goedecker, M. Teter, and J. Hutter, Phys. Rev. B 54, 1703 (1996).

${ }^{18}$ G.B. Bachelet, D.R. Hamann, and M. Schlüter, Phys. Rev. B 26, 4199 (1982). 